\title{
Eficiencia energética con Generación Distribuida Fotovoltaica (GD-PV); Caso de una Congeladora de Pescados y Mariscos en Campeche, México
}

\section{Energy efficiency using Distributed Generation; a Fish and Seafood Freezer case in Campeche, Mexico}

CHAN-GONZALEZ, Jorge J.†*, SHIH, Meng Yen, LEZAMA-ZÁRRAGA, Francisco, NOH-PAT, Felipe

Universidad Autónoma de Campeche, Campus V, Predio s/n por Av. Humberto Lanz Cárdenas y Unidad Habitacional Ecológica Ambiental, Col. Ex-Hacienda Kalá, CP 24085, San Francisco de Campeche, Cam., México

ID $1^{\text {er }}$ Autor: Jorge J, Chan-Gonzalez / ORC ID: 0000-0002-8638-1646, CVU CONACYT ID: 89415

ID 1 ${ }^{\mathrm{er}}$ Coautor: Meng Yen, Shih / ORC ID: 0000-0001-7475-6458, CVU CONACYT ID: 408617

ID $2^{\text {do }}$ Coautor: Francisco, Lezama-Zárraga / ORC ID: 0000-0003-3397-7881, Researcher ID Thomson: U-1229-2018, CVU CONACYT ID: 205493

ID $3^{\text {er }}$ Coautor: Felipe, Noh-Pat / ORC ID: 0000-0003-1981-8323, CVU CONACYT ID: 260889

DOI: $10.35429 /$ JRE.2020.12.4.21.31

Recibido 09 de Abril, 2020; Aceptado 30 Junio, 2020

\section{Resumen}

En este artículo se presenta un estudio de eficiencia energética a una empresa congeladora de pescados y mariscos que posee una planta de Generación Distribuida Fotovoltaica (GD-PV) con una capacidad instalada de 60.32 kWp; se realizó una auditoría energética, se analizó la información de las principales variables eléctricas que actúan como protagonistas en la facturación del servicio de la empresa suministradora. La Generación Distribuida GD a partir de energías renovables se ha ido incrementado a nivel mundial como un medio eficaz para reducir los costos de la energía eléctrica y que además ayuda directamente a que las empresas sean más competitivas y favorecer el crecimiento económico. La Generación Distribuida Fotovoltaica (GD-PV) proporciona otros beneficios adicionales como la mitigación de gases de efecto invernadero y funcionar como una barrera en los techos que impide la ganancia de radiación solar en los edificios; y este hecho es particularmente relevante en un clima cálido-húmedo como lo tiene el estado de Campeche, México. El área sombreada por la planta GD$\mathrm{PV}$, evita que haya una ganancia térmica por techo, diaria de aproximadamente $236.62 \mathrm{~kW}$. Con la planta GD-PV se evita la emisión a la atmosfera de 4,448.9 $\mathrm{kg}$ de $\mathrm{CO}_{2}$ equivalente.

Generación distribuida, Eficiencia energética, Sistemas fotovoltaicos interconectados a red, Generación distribuida fotovoltaica

\begin{abstract}
In this article, an energy efficiency study is presented for the fish and seafood freezer company which possesses a Photovoltaic Distributed Generation (GD-PV) plant of $60.32 \mathrm{kWp}$ installed capacity. An energy audit is performed which consists of analyzing the principal electric variables for the utility billing service. Distributed Generation (GD) from renewable energy has been increasing worldwide as an effective mean to reduce electrical energy costs. It also directly helps companies to be more competitive and promote economic growth. Photovoltaic Distributed Generation (GD-PV) provides other additional benefits such as greenhouse gas mitigation and functioning as a roof barrier that prevents gaining solar radiation in buildings. This fact is particularly relevant in a hot-humid climate such as the state of Campeche, Mexico. The shading area due to GD$\mathrm{PV}$ prevents a daily roof thermal gaining of approximately $236.62 \mathrm{~kW}$. Moreover, the GD-PV prevents the atmospheric emission of $4,448.9 \mathrm{~kg}$ of $\mathrm{CO}_{2}$ equivalent.
\end{abstract}

Distributed generation, Energy efficiency, Gridconnected photovoltaic systems, Photovoltaic distributed generation 


\section{Introducción}

La Generación Distribuida GD a partir de energías renovables se ha ido incrementado a nivel mundial como un medio eficaz para reducir los costos de la energía eléctrica y que además ayuda directamente a que las empresas sean más competitivas y favorecer el crecimiento económico. La Generación Distribuida Fotovoltaica (GD-PV) proporciona otros beneficios adicionales como la mitigación de gases de efecto invernadero y funcionar como una barrera en los techos que impide la ganancia de radiación solar en los edificios y este hecho es particularmente relevante en un clima cálidohúmedo como lo tiene el estado de Campeche, México; y aún más cuando la GD-PV se está realizando en una empresa congeladora de pescados y mariscos y que uno de los mayores retos a vencer es el impedir las ganancias de calor, éstos y otros fundamentos pueden generar beneficios económicos netos para justificar el pago de la inversión inicial. Según la Ley de la Industria Eléctrica y el Manual de interconexión de Centrales de GD con capacidad menor a 0.5 MW, la GD se define como la generación de energía eléctrica que se encuentra interconectada a un circuito de distribución que contenga una alta concentración de Centros de Carga (CRE 2017).

La GD incluye aquella que se realiza por un generador exento, en otras palabras, el propietario de una o más centrales eléctricas con capacidad menor a $0.5 \mathrm{MW}$ que no requieren permiso para generar energía eléctrica. La GD puede ser localizada en las instalaciones de los Centros de Carga o fuera de éstos (SENER, 2016). Se espera, a nivel mundial, que la energía solar fotovoltaica distribuida, se establezca en más del doble de su capacidad en los próximos cinco años, lo que representa casi la mitad de todo el crecimiento de la energía solar fotovoltaica.

Los sistemas GD-PV en hogares, edificios comerciales e industria arrojarán cambios sustantivos en los sistemas energéticos. Este rápido aumento en la capacidad que tendrán los consumidores en generar su propia electricidad, representa un nicho de oportunidades para los proveedores del servicio y los garantes políticos en todo el mundo.

\section{Justificación}

La energía eléctrica representa para las pequeñas y medianas industrias en México uno de los costos más elevados durante sus procesos de producción. En términos simples, en el año 2019 el costo de electricidad era el 5 por ciento de los ingresos de un hotel; ahora es del 15 por ciento. (Asociación Mexicana de Hoteles y Moteles)

Para el caso mexicano, en los últimos años los contratos de GD-PV han aumentado de manera importante. En 2017 se concretaron 29,676 contratos y en 2018 fueron 35,661; esto representa una tasa de crecimiento media anual de aproximadamente 20\%. En capacidad representaron 211.65 y 233.56 MW, respectivamente (Monitor de información comercial e índice de precios de Generación Solar Distribuida en México, 2019). Figura 1.

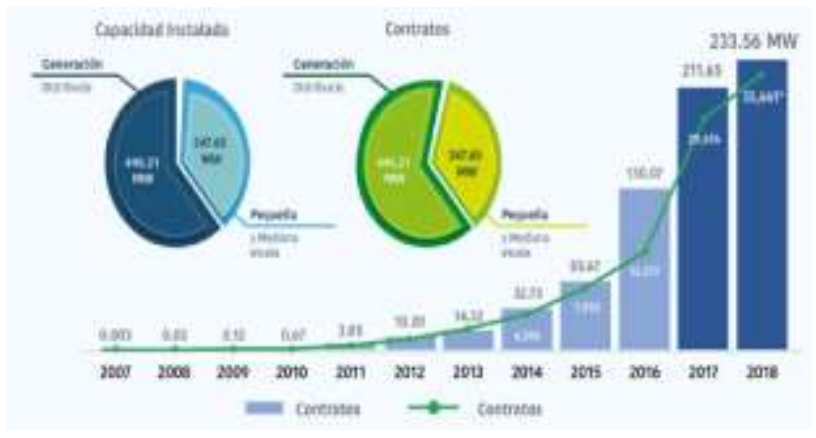

Figura 1 Evolución de contratos y capacidad instalada en México

Fuente: Monitor de información comercial e índice de precios de Generación Solar Distribuida en México

De esta misma fuente, de 2017 al primer semestre del año 2019, se presentan los estados que instalaron las mayores cantidades de GD-PV donde es muy claro que los estados de la república mexicana que mayor capacidad instalada acumulada son Jalisco, Nuevo León, CDMX, Chihuahua y Yucatán, figura 2. Observamos también de la figura 2 que el estado de Campeche posee una capacidad instalada acumulada muy pequeña, menor de $5 \mathrm{MW}$. Sin embargo, el estado de Campeche posee buen recurso de radiación solar; se cuenta con una irradiación que va de 4.5 a $6 \mathrm{kWh} / \mathrm{m}^{2}$ al día. Datos proporcionados por NREL. 


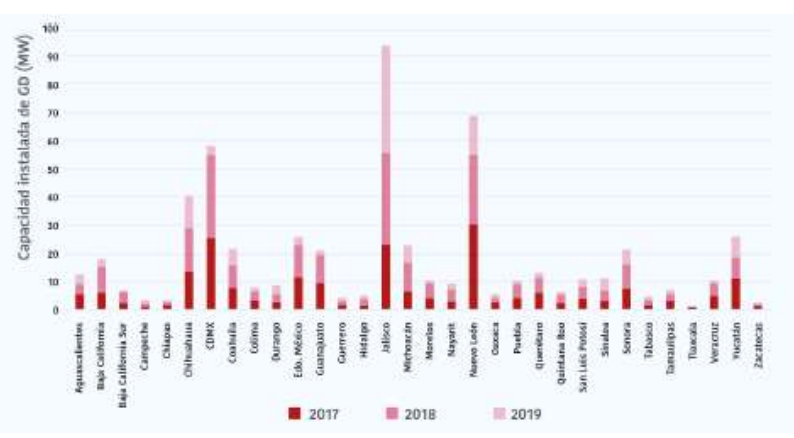

Figura 2 Evolución de la capacidad de GD-PV en México por estado

Fuente: Monitor de información comercial e índice de precios de Generación Solar Distribuida en México

Esta condición en Campeche de poseer buena irradiancia, necesidad energética y una pequeña capacidad instalada acumulada nos lleva a concluir que se abre un nicho de oportunidades importante en la instalación de Sistemas de generación distribuidas fotovoltaicas.

En adición, Campeche es un estado con clima húmedo-cálido; se ve en la necesidad de emplear grandes cantidades de energía eléctrica para los procesos de refrigeración (alta, media y baja temperatura). En el reporte que emite la Universidad Autónoma de Campeche a la Secretaria de Desarrollo Económico del estado de Campeche en 2019, indica que al menos el $60 \%$ del consumo eléctrico en casas-habitación, pequeñas y medianas industrias, Escuelas y Universidades, es empleado para los procesos de refrigeración en cualquiera de sus modalidades.

En ese sentido, un aspecto que se vuelve muy relevante es evitar la excesiva ganancia de calor en los espacios a refrigerar y particularmente evitar la ganancia de calor por radiación solar en techos y muros. Se sabe (Victorio Santiago Díaz et al, 2005) que aproximadamente de 50 a $60 \%$ de la ganancia total es debido a la radiación solar.

En aplicaciones de ingeniería en acondicionamiento de aire, se puede considerar una ganancia solar promedio por techo de 550 $\mathrm{w} / \mathrm{m}^{2}$, como la energía captada en un metro cuadrado de superficie, en posición horizontal con el sol en el cenit y considerando una atmósfera limpia con un grado de turbiedad bajo. Podemos inferir que un valor agregado de los sistemas GD-PV, en los climas cálidoshúmedos, e instalados en los techos es que evitan la ganancia de calor por radiación solar en los edificios.
Como consecuencia se requiere de menor energía eléctrica para los procesos de refrigeración. De esta forma se promueve la eficiencia energética.

Con lo que respecta a la eficiencia energética se tiene que de acuerdo con Deutsche Gesellschaft fur Internationale Zusammenarbeit (GIZ-2014) en su guía de buenas prácticas para el ahorro y uso eficiente de la energía la define como: la obtención de los mismos bienes y servicios energéticos, pero con mucha menos energía, con la misma o mayor calidad de vida, con menos contaminación, a un precio inferior al actual, alargando la vida de los recursos y con menos conflicto

\section{Objetivo}

Demostrar que con al implementar Generación Distribuida con sistemas fotovoltaicos (GD-PV) se logra la eficiencia energética en una empresa congeladora de pescados y mariscos.

\section{Hipótesis}

Al implementar una planta GD-PV en una empresa congeladora se logra eficiencia energética y disminución de sus costos de producción; así como se evita la emisión a la atmosfera de $\mathrm{CO}_{2}$ equivalente.

\section{Planteamiento del problema}

Se desea obtener la disminución del consumo energético de una planta congeladora de Pescados y Mariscos ubicada en el estado de Campeche. El estado de Campeche se encuentra ubicado geográficamente en la Península de Yucatán entre los paralelos $17^{\circ} 49^{\prime}$ y $20^{\circ} 51^{\prime}$ latitud norte; y entre los meridianos $89^{\circ} 06^{\prime}$ y $92^{\circ} 27^{\prime}$ oeste; El proceso mismo de congelación demanda altos valores de energía para poder congelar los productos que comercializa. Las condiciones ambientales en el lugar son factores importantes adversos durante éste proceso. $\mathrm{La}$ ubicación de la planta está $19^{\circ} 38^{\prime} 22^{\prime \prime} \mathrm{N}$ $90^{\circ} 41^{\prime} 18^{\prime \prime} \mathrm{O}$ a 20 metros de la costa con una temperatura promedio de $31 \pm 0.1^{\circ} \mathrm{C}$ y una humedad relativa de $75 \pm 1 \%$, también promedio (INEGI, 2015), al nivel del mar. La empresa cuenta con 6 unidades de congelamiento de 7 toneladas de refrigeración (TR) ó $12 \mathrm{~kW}$, cada una, es decir son $42 \mathrm{TR}$ (72 kW); estos equipos son los que presentan la mayor demanda de energía. 
También cuenta con una pequeña fábrica de hielo a escamas, de $9.3 \mathrm{~kW}$. Tienen instalados otros equipos de menor capacidad como aires acondicionados tipo mini Split de una tonelada de refrigeración, iluminación y equipos misceláneos. Dada esta situación de demanda energética se planteó la posibilidad de instalar una planta de Generación Distribuida Fotovoltaica (GD-PV).

\section{Descripción del Sistema de generación Distribuida Fotovoltaica (GD-PV).}

Se trata de una planta generadora de energía eléctrica producida con módulos fotovoltaicos. Está constituida por 3 páneles fotovoltaicos compuestos, cada uno, de 5 cadenas de doce módulos de $260 \mathrm{Wp}$ cada uno, conectados en serie y un panel de 4 cadenas con trece módulos (de las mismas características descritas anteriormente). Cada panel tiene su propio inversor. Son tres inversores de $15.6 \mathrm{kWp}$ y uno de $13.5 \mathrm{kWp}$. En total son 232 módulos fotovoltaicos, con una capacidad instalada total de $60.32 \mathrm{kWp}$, figura 3. Cada módulo fotovoltaico tiene un área de $2 \mathrm{~m}^{2}$.

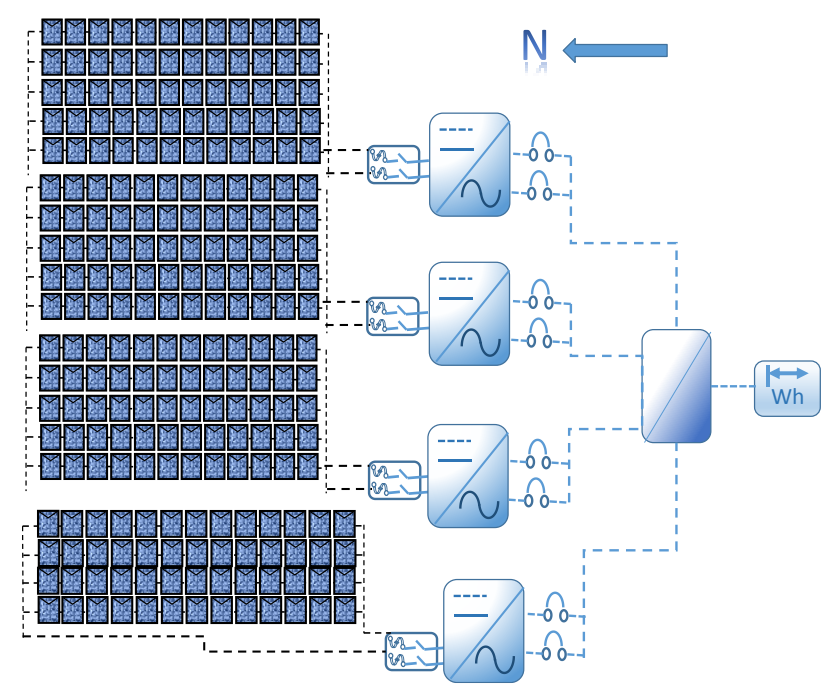

Figura 3 Diagrama esquemático de la planta de Generación Distribuida Fotovoltaica. Consta de 3 páneles $15.6 \mathrm{kWp}$ y un pánel de $13.5 \mathrm{kWp}$

Fuente: Elaboración Propia

La instalación fotovoltaica tiene una inclinación de 22 grados con respecto al plano horizontal; por lo que resulta que cada módulo sombrea un área de $1.85 \mathrm{~m}^{2}$, en otras palabras, por toda la instalación fotovoltaica se produce un área de techo sombreada de aproximadamente $430 \mathrm{~m}^{2}$.
La planta fotovoltaica se encuentra orientada totalmente hacia el sur, es correcta su declinación azimutal. Se observa que los módulos fotovoltaicos en su totalidad, están colocados prácticamente a ras de techo, lo que provoca que no exista circulación de aire entre los módulos, provocando que éstos se calienten de más, disminuyendo considerablemente sus eficiencias de producción (Agustín Castejón2012). Figura 4.

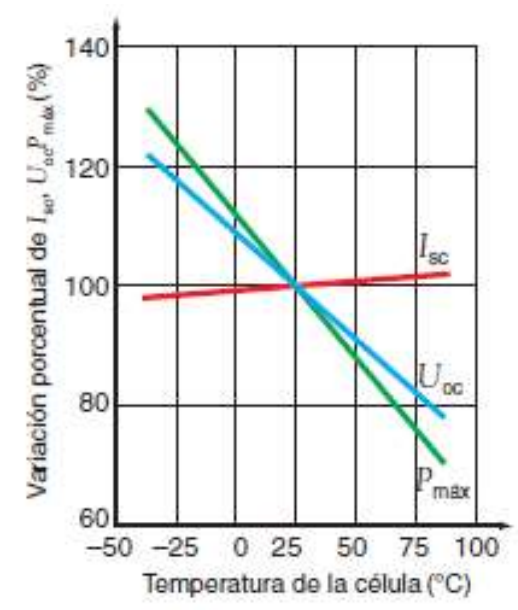

Figura 4 Variación porcentual del voltaje a circuito abierto, corriente de cortocircuito y la potencia máxima de un módulo fotovoltaico con respecto a la temperatura Fuente: Castejón 2012

Por otra parte el techo de la congeladora tiene una barda perimétrica de un metro de alto y tampoco favorece una buena circulación del aire; por lo mismo no se favorece un buen enfriamiento de los módulos fotovoltaicos. Figura 5.

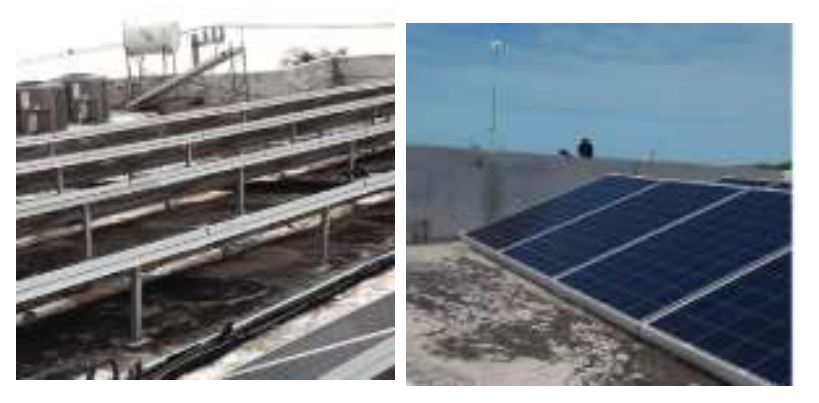

Figura 5 Vista parcial de la planta de Generación Distribuida Fotovoltaica (GD-PV) en Campeche. Se aprecia una barda perimetral y la nula separación de los módulos al techo, evitando el buen enfriamiento de los módulos Fuente: Elaboración Propia

Rayenari D. C. et al, (2015) recomiendan que para que la instalación fotovoltaica tenga una mejor eficiencia en épocas pico (de marzo a agosto), se reajuste la inclinación a 10 o 15 grados. 
Se puede ganar hasta $5 \%$ de eficiencia y se evita esfuerzos mecánicos a los módulos por su propio peso y por la nula circulación de viento (zona de estancamiento) Potter et al (2011). De igual forma se recomienda levantar los módulos, tomando como referencia la parte más baja de los módulos, por lo menos cincuenta centímetros con respecto del techo. Favorecerá la circulación de aire para enfriar los módulos y se evitará zonas de estancamiento. Se prevé un aumento de eficiencia global de un $5 \%$.

\section{Modelado Matemático de la ganancia de calor por el techo de la congeladora}

De acuerdo con Víctor Lanz G. et al (2018), se tiene documentado que en climas cálidos húmedos la ganancia de calor en los techos de las edificaciones representa el $40 \%$ del calor recibido por un edificio a lo largo del día. Por lo que se hace muy importante cuantificar la ganancia de calor por techos. Se tienen las siguientes ecuaciones para cuantificar las ganancias de calor por techo, como un efecto combinado de conducción, convección y radiación (Céngel et al-2012).

$$
\begin{gathered}
\dot{Q}=\dot{Q}_{\text {habi-techo,conv }+ \text { rad }}=\dot{Q}_{\text {cond }, \text { techo }} \\
=\dot{Q}_{\text {techo-amb,cond }+ \text { rad }} \\
\dot{Q}_{\text {habi-techo,conv }+ \text { rad }}= \\
h_{i} A\left(T_{\text {habi }}-T_{s, \text { int }}\right)+\varepsilon A \sigma\left(T_{\text {habit }}^{4}-T_{s, \text { int }}^{4}\right) \\
\dot{Q}_{\text {cond,techo }}=k A \frac{T_{s, \text { int }}-T_{s, \text { ext }}}{L} \\
\dot{Q}_{\text {techo-alrededor,cond }+ \text { rad }}= \\
h_{o} A\left(T_{s, \text { ext }}-T_{\text {amb }}\right) \\
+\varepsilon A \sigma\left(T_{s, \text { ext }}^{4}\right. \\
\left.-T_{a m b}^{4}\right)-\alpha \dot{q}_{\text {solar }}
\end{gathered}
$$

Donde $\dot{Q}$ es el flujo de calor total, $k$ conductividad térmica, $\alpha$ absortividad, $h$ coeficiente de transferencia de calor convectivo, $\sigma$ la constante de Steffan-Boltzman, $A$ área de transferencia de calor, $\dot{q}_{\text {solar }}$ radiación solar y $T$ temperatura. Se obtiene que por cada metro cuadrado de techo se recibe una radiación de 550 $\mathrm{w} / \mathrm{m}^{2 *}$ día, y se tienen aproximadamente $430 \mathrm{~m}^{2}$ de área sombreada por la planta fotovoltaica, entonces se puede estimar que se evita que haya una ganancia térmica por techo diaria de aproximadamente $236.62 \mathrm{~kW}$.
Como referencia los equipos de acondicionamiento de aire tipo mini Split de una tonelada de refrigeración tienen una capacidad térmica de retirar calor de $3.54 \mathrm{kWh}$. Por lo tanto, antes de instalar la GD-PV la ganancia por calor por techos era muy elevada para el edificio en conjunto.

\section{Metodología para el análisis de la eficiencia energética en los procesos de la empacadora}

En el análisis de la eficiencia energética de la congeladora, fue necesario conocer la dinámica

\begin{tabular}{|c|c|c|c|}
\hline Parámetro & Intervalo & Resolución & Precisión \\
\hline $\begin{array}{l}\text { Vrms } \\
(\mathrm{CA}+\mathrm{CC})\end{array}$ & $\begin{array}{l}1 \mathrm{~V} \text { a } 1000 \\
\mathrm{~V} \text { fase a } \\
\text { neutro }\end{array}$ & $0,1 \mathrm{~V}$ & $\begin{array}{l} \pm 0,5 \% \text { de } \\
\text { la tensión } \\
\text { nominal }\end{array}$ \\
\hline $\begin{array}{ll}\text { Pico } & \mathrm{de} \\
\text { tensión } & \end{array}$ & \begin{tabular}{lr}
\multicolumn{2}{l}{$1 \mathrm{~V}$ pico de } \\
tensión & a \\
1400 & $\mathrm{~V}$ \\
pico & de \\
tensión & \\
\end{tabular} & $1 \mathrm{~V}$ & $\begin{array}{l}5 \% \text { de la } \\
\text { tensión } \\
\text { nominal }\end{array}$ \\
\hline 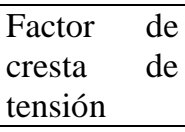 & & $1,0>2,8$ & $0,01 \pm 5 \%$ \\
\hline $\mathrm{Vrms}^{1 / 2}$ & $\begin{array}{l}1 \mathrm{~V} \text { a } 1000 \\
\mathrm{~V} \text { fase a } \\
\text { neutro }\end{array}$ & $0,1 \mathrm{~V}$ & $\begin{array}{l} \pm 1 \% \text { de la } \\
\text { tensión } \\
\text { nominal }\end{array}$ \\
\hline $\mathrm{V}_{\text {fund }}$ & $\begin{array}{l}1 \mathrm{~V} \text { a } 1000 \\
\mathrm{~V} \text { fase a } \\
\text { neutro }\end{array}$ & $0,1 \mathrm{~V}$ & $\begin{array}{l} \pm 0,5 \% \text { de } \\
\text { la tensión } \\
\text { nominal }\end{array}$ \\
\hline $\begin{array}{l}\text { Amperios } \\
(\mathrm{CA}+\mathrm{CC})\end{array}$ & $\begin{array}{l}0,5 \text { A a } 600 \\
\text { A }\end{array}$ & $0,1 \mathrm{~A}$ & $\begin{array}{l} \pm 0,5 \% \pm \\
5 \text { cuentas }\end{array}$ \\
\hline Frecuencia & $\begin{array}{l}51,00 \mathrm{~Hz} \mathrm{a} \\
69,00 \mathrm{~Hz}\end{array}$ & $0,01 \mathrm{~Hz}$ & $\pm 0,01 \mathrm{~Hz}$ \\
\hline $\begin{array}{l}\text { kWh kVAh, } \\
\text { kvarh }\end{array}$ & & & $\begin{array}{l} \pm 1 \% \pm 10 \\
\text { cuentas }\end{array}$ \\
\hline $\begin{array}{l}\text { Factor de } \\
\text { cresta } \\
\text { de amperios }\end{array}$ & 5 A a 6000 & $1 \mathrm{~A}$ & $\begin{array}{c} \pm 1 \% \pm \\
10 \text { cuentas }\end{array}$ \\
\hline Amps $1 / 2$ & $\begin{array}{l}5 \text { A a } 6000 \\
\text { A }\end{array}$ & $1 \mathrm{~A}$ & $\begin{array}{l} \pm 0.5 \% \pm \\
5 \text { cuentas }\end{array}$ \\
\hline $\mathrm{A}_{\text {fund }}$ & $\begin{array}{l}5 \text { A a } 6000 \\
\text { A }\end{array}$ & $1 \mathrm{~A}$ & $\begin{array}{l} \pm 0.1 \% \pm \\
5 \text { cuentas }\end{array}$ \\
\hline
\end{tabular}
de los parámetros eléctricos involucrados en los procesos de funcionamiento de la Planta, por lo cual, realizamos un diagnóstico energético. Para este propósito se instaló un equipo analizador de la calidad de la energía y potencia eléctrica, trifásica marca FLUKE, modelo 430 Serie II, figura 6. Se muestran sus características técnicas en la tabla 1.

Tabla 1 Parámetros, intervalos de funcionamiento, resolución y precisión del equipo analizador de la calidad de la energía y potencia eléctrica, trifásico marca FLUKE, modelo 430 Serie II

Fuente Elaboración Propia 


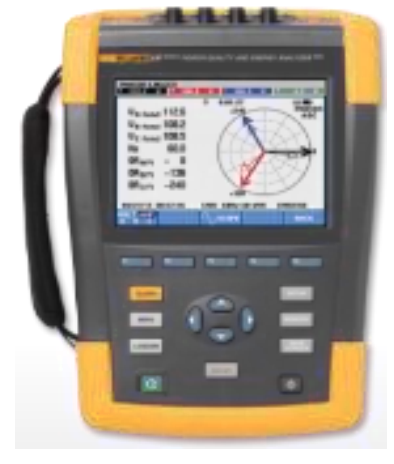

Figura 6 Analizador de la calidad de la energía y potencia eléctrica, trifásico marca FLUKE, modelo 430 Serie II Fuente: Elaboración Propia

\section{Metodología propuesta para la auditoría energética}

De acuerdo con Lezama et al (2018), la metodología propuesta consta de las siguientes etapas:

1. Planeación. Consiste en programa las visitas al lugar, la preparación de materiales, herramientas, equipo de medición y del personal que realizará el trabajo de campo.

2. Investigación in situ. Acudir a las instalaciones de acuerdo con lo calendarizado para iniciar la inspección con ayuda de los planos eléctricos de cada área de trabajo y conectar el analizador de redes en la sección de la instalación o equipo que se desea estudiar

3. Recopilación de la información. Realizar el levantamiento físico en las instalaciones mediante censo de carga ( $\mathrm{kW}$ instalados). Asimismo, reunir los datos de consumos $(\mathrm{kWh})$, demandas $(\mathrm{kW})$ y factor de potencia (f.p.) mensuales durante un año a través de la factura de la compañía suministradora de energía eléctrica. En adición, extraer del analizador de calidad de la energía y potencia la información de consumos $(\mathrm{kWh})$, demandas $(\mathrm{kW})$, tensiones (Volts), corrientes (Amperes), factor de potencia (f.p.) y frecuencia $(\mathrm{Hz})$ por cada fase y totales.

4. Análisis de la información. Analizar la información recopilada a través de hojas de cálculo, con tablas y gráficas que permitan describir en qué condiciones se encuentra funcionando el sistema eléctrico bajo estudio o si ocurre un fenómeno que lo esté perturbando.
5. Definir las propuestas de mejora que optimizarán los procesos internos a través del ahorro y uso eficiente de la energía eléctrica.

6. Informe final. Realizar la Memoria Técnica Descriptiva del diagnóstico energético con las propuestas de ahorro y uso eficiente de la energía.

En la siguiente sección se presenta el análisis de la información recabada la cual nos proporcionará el comportamiento de las variables eléctricas de la instalación y de esta manera realizar propuestas de ahorro y uso eficiente de energía para la optimización de los procesos de la Planta congeladora.

\section{Análisis de la información y resultados obtenidos}

Luego de aplicar los primeros tres pasos de la metodología propuesta, el análisis de la información se realiza en hojas de cálculo Excel. Iniciamos con el análisis de las facturas de energía eléctrica mostradas en la tabla 2 , en la que se reúne la información de consumos (kWh), demandas (kW) y factor de potencia (f.p.) mensuales durante un año obtenida de la factura de energía eléctrica que emite la compañía suministradora del servicio (en México es la Comisión Federal de Electricidad, CFE).

\begin{tabular}{|l|r|r|r|}
\hline \multicolumn{1}{|c|}{ Periodo } & \multicolumn{1}{c|}{$\begin{array}{c}\text { Demanda } \\
\text { máxima } \\
\text { (kW) }\end{array}$} & $\begin{array}{c}\text { Consumo } \\
\text { total } \\
\text { (kWh) }\end{array}$ & $\begin{array}{c}\text { Factor de } \\
\text { potencia } \\
(\%)\end{array}$ \\
\hline Ene-19 & 82 & 35,520 & 82.15 \\
\hline Feb-19 & 73 & 31,680 & 81.25 \\
\hline Mar-19 & 83 & 30,560 & 81.27 \\
\hline Abr-19 & 106 & 40,400 & 81.41 \\
\hline May-19 & 85 & 33,360 & 80.85 \\
\hline Jun-19 & 98 & 41,360 & 82.44 \\
\hline Jul-19 & 83 & 32,640 & 81.08 \\
\hline Ago-19 & 86 & 34,000 & 81.61 \\
\hline Sep-19 & 76 & 31,040 & 81.33 \\
\hline Oct-19 & 76 & 29,760 & 81.42 \\
\hline Nov-19 & 84 & 32,880 & 82.15 \\
\hline Dic-19 & 78 & 31,600 & 79.90 \\
\hline Ene-20 & 80 & 35,863 & 82,19 \\
\hline
\end{tabular}

Tabla 2 Análisis del historial de las facturaciones mensuales de la Congeladora

Fuente: Elaboración Propia

Se observa en la Tabla 2 que la mayor facturación se presenta en el mes de abril debido a las altas temperaturas ambientales y que la potencia instalada demanda mayor energía, principalmente en refrigeración y aire acondicionado.

CHAN-GONZALEZ, Jorge J., SHIH, Meng Yen, LEZAMAZÁRRAGA, Francisco, NOH-PAT, Felipe. Eficiencia energética con Generación Distribuida Fotovoltaica (GD-PV); Caso de una Congeladora de Pescados y Mariscos en Campeche, México. Revista de Energías Renovables. 2020 


\section{Levantamiento o censo de cargas}

Del levantamiento o censo de cargas, se obtiene la potencia instalada, la cual es la suma de la potencia en $\mathrm{kW}$ de todos los equipos eléctricos conectados a la instalación. El censo de carga se presenta en un cuadro de cargas, en el cual se determina el \% de desbalance entre las tres fases. La tabla 3 presenta un cuadro de cargas general de toda la carga instalada y en cual fase está conectada cada carga.

\begin{tabular}{|c|c|c|c|c|}
\hline Carga & $\begin{array}{c}\mathbf{k W} \\
\text { fase A }\end{array}$ & $\begin{array}{c}\mathbf{k W} \\
\text { fase B }\end{array}$ & $\begin{array}{c}\mathbf{k W} \\
\text { fase } \mathbf{C}\end{array}$ & $\begin{array}{l}\text { kW } \\
\text { total }\end{array}$ \\
\hline $\begin{array}{lcc}6 & \text { Unidades } & \text { de } \\
\text { congelamiento } & \end{array}$ & 24 & 24 & 24 & 72 \\
\hline 1 Fábrica de hielo & 3.1 & 3.1 & 3.1 & 9.3 \\
\hline 3 Minisplits de 1TR & 3.6 & 3.6 & 3.6 & 10.8 \\
\hline Miscelanea & 1.8 & 1.2 & 1.8 & 4.8 \\
\hline Alumbrado & 2.7 & 2.1 & 3.6 & 8.4 \\
\hline Contactos & 1.6 & 0.8 & 1.8 & 4.2 \\
\hline Total & 36.8 & 34.8 & 37.9 & 109.5 \\
\hline & & $\begin{array}{l}\text { Potenci } \\
\text { instalad }\end{array}$ & total & 109.5 \\
\hline
\end{tabular}

Tabla 3 Potencia total instalada en el sistema eléctrico de la Congeladora

Fuente: Elaboración Propia

De acuerdo con la NOM-001-SEDE-2012, el porcentaje de desbalance permitido entre fases en un sistema $3 \mathrm{~F}-4 \mathrm{H}$ no debe exceder al 5\%. Al aplicar la ecuación (5),

$$
\% \text { Desbalance }=\frac{k W_{\text {mayor }}-k W_{\text {menor }}}{k W_{\text {mayor }}} * 100
$$

se verifica que

$\%$ Desbalance $=\frac{37.9 \mathrm{~kW}-34.8 \mathrm{~kW}}{37.9 \mathrm{~kW}} * 100=8.17$

éste es un porcentaje mayor a lo permitido, por lo que se tuvo que realizar un análisis para reducir el \% desbalance en el sistema eléctrico de la Planta congeladora.

A continuación, se hace la propuesta para reducir el \% desbalance cambiando algunas cargas monofásicas a otra fase. Esta propuesta se muestra en la tabla 4 y se comprueba que el porcentaje de desbalance obtenido se reduce y es aceptable por la NOM-001-SEDE-2012. En la tabla 4 se muestran los cambios de carga a través del intercambio de fase que se proponen con las celdas marcadas en color azul.

\begin{tabular}{|c|c|c|c|c|}
\hline Carga & $\begin{array}{c}\mathbf{k W} \\
\text { fase } \mathbf{A}\end{array}$ & $\begin{array}{c}\mathbf{k W} \\
\text { fase } B\end{array}$ & $\begin{array}{c}\mathbf{k W} \\
\text { fase } \mathbf{C}\end{array}$ & $\begin{array}{l}\mathbf{k W} \\
\text { total }\end{array}$ \\
\hline $\begin{array}{l}6 \text { Unidades de } \\
\text { congelamiento }\end{array}$ & 24 & 24 & 24 & 72 \\
\hline $\begin{array}{l}1 \text { Fábrica de } \\
\text { hielo }\end{array}$ & 3.1 & 3.1 & 3.1 & 9.3 \\
\hline $\begin{array}{l}3 \text { Minisplits de } \\
\text { 1TR }\end{array}$ & 3.6 & 3.6 & 3.6 & 10.8 \\
\hline Miscelanea & 1.8 & 1.8 & 1.2 & 4.8 \\
\hline Alumbrado & 2.7 & 2.1 & 3.6 & 8.4 \\
\hline Contactos & 0.8 & 1.6 & 1.8 & 4.2 \\
\hline Total & 36 & 36.2 & 37.3 & 109.5 \\
\hline & & $\begin{array}{l}\text { Potencic } \\
\text { instalad }\end{array}$ & total & 109.5 \\
\hline
\end{tabular}

Tabla 4 Potencia total instalada en el sistema eléctrico de la Congeladora. Se observa en color azul los cambios de carga en las fases para disminuir el \% desbalance Fuente: Elaboración Propia

De acuerdo con la tabla anterior, tenemos:

$\%$ Desbalance $=\frac{37.3 \mathrm{~kW}-36.2 \mathrm{~kW}}{37.3 \mathrm{~kW}} * 100=3.48$

y este $\%$ desbalance es aceptado por la NOM-001-SEDE-2012.

\section{Resultados del analizador de calidad de la energía y potencia eléctrica}

La información obtenida de las mediciones fue analizada en hoja de cálculo Excel. Cada 10 minutos; el analizador de calidad de la energía (ver tabla 1) y potencia eléctrica guarda la información de las variables eléctricas de los datos medidos, siendo en total 1007 muestras por cada variable durante los ocho días que estuvo conectado al sistema eléctrico de la Planta Congeladora. La potencia máxima por día que demanda la instalación se muestra en la figura 7 . Se observa que la mayor demanda se presenta en un horario nocturno, entre las 18:00 y las 21:00 horas, en el que el GD-PV no aporta potencia, por lo que la instalación la toma de la red de la CFE.

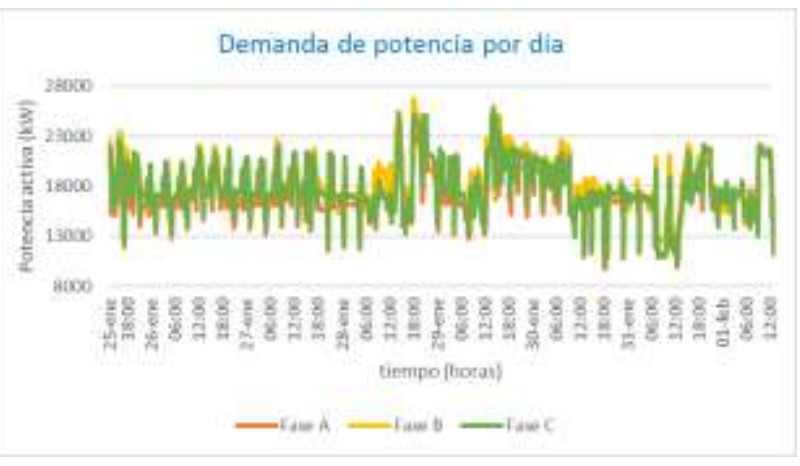

Figura 7 Potencia máxima por día que demanda la instalación eléctrica de la congeladora

Fuente: Elaboración Propia

CHAN-GONZALEZ, Jorge J., SHIH, Meng Yen, LEZAMAZÁRRAGA, Francisco, NOH-PAT, Felipe. Eficiencia energética con Generación Distribuida Fotovoltaica (GD-PV); Caso de una Congeladora de Pescados y Mariscos en Campeche, México. Revista de Energías Renovables. 2020 
La Tabla 5 ilustra la demanda máxima de cada día, en que se verifica que la medición que registra el medidor bidireccional de CFE para la facturación del mes es de $76.69 \mathrm{~kW}$.

\begin{tabular}{|l|r|r|r|r|}
\hline Fecha & $\begin{array}{c}\text { kWmáx } \\
\text { /día } \\
\text { Fase A }\end{array}$ & \multicolumn{1}{c|}{$\begin{array}{c}\text { kWmáx/día } \\
\text { Fase B }\end{array}$} & $\begin{array}{c}\text { kWmáx/día } \\
\text { Fase C }\end{array}$ & $\begin{array}{c}\text { kWmáx/día } \\
\text { Total }\end{array}$ \\
\hline 25-ene & 22.32 & 23.49 & 22.69 & 68.5 \\
\hline 26-ene & 21.37 & 22.18 & 21.8 & 65.24 \\
\hline 27-ene & 21 & 22.67 & 21.91 & 65.54 \\
\hline 28-ene & 25.41 & 26.7 & 25.14 & 76.02 \\
\hline 29-ene & 25.51 & 25.29 & 25.89 & 76.69 \\
\hline 30-ene & 21.04 & 22.62 & 21.36 & 64.68 \\
\hline 31-ene & 21.65 & 22.3 & 22.18 & 65.37 \\
\hline 01-feb & 22.02 & 21.81 & 22.11 & 65.84 \\
\hline
\end{tabular}

Tabla 5 Demanda máxima durante los días de medición en el sistema eléctrico de la Congeladora

Fuente: Elaboración Propia

\begin{tabular}{|l|r|r|r|r|}
\hline \multicolumn{2}{|r|}{ Fecha } & $\begin{array}{c}\text { kWh/dí } \\
\text { a Fase } \\
\text { A }\end{array}$ & \multicolumn{1}{c|}{$\begin{array}{c}\text { kWh/día } \\
\text { Fase B }\end{array}$} & \multicolumn{2}{c|}{$\begin{array}{c}\text { kWh/día } \\
\text { Fase C }\end{array}$} & $\begin{array}{c}\text { kWh/día } \\
\text { Total }\end{array}$ \\
\hline 25-ene & 412.586 & 412.586 & 412.586 & 1237.758 \\
\hline 26-ene & 408.268 & 434.27 & 426.821 & 1269.359 \\
\hline 27-ene & 412.552 & 441.682 & 434.008 & 1288.242 \\
\hline 28-ene & 425.193 & 460.211 & 437.593 & 1322.997 \\
\hline 29-ene & 435.247 & 471.07 & 453.865 & 1360.182 \\
\hline 30-ene & 399.014 & 424.189 & 408.864 & 1232.068 \\
\hline 31-ene & 386.912 & 409.476 & 394.205 & 1190.592 \\
\hline 01-feb & 420.762 & 445.31 & 428.693 & 1294.765 \\
\hline
\end{tabular}

Tabla 6 Consumo total durante los días de medición en el sistema eléctrico de la Congeladora

Fuente: Elaboración Propia

La energía consumida por el sistema eléctrico durante el período de medición se muestra en la Tabla 6 y en la figura 8 . Se observa un consumo casi uniforme durante los ocho días de medición.

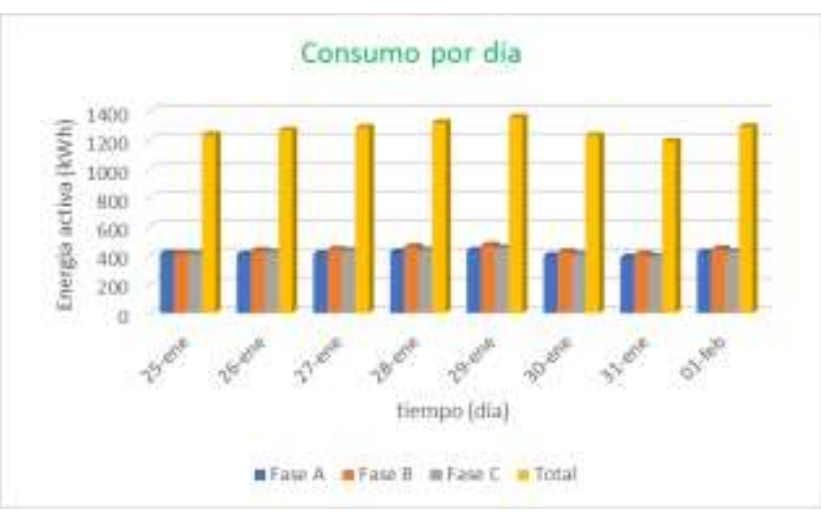

Figura 8 Consumo total, presentado en barras, durante los días de medición en el sistema eléctrico de la Congeladora Fuente: Elaboración Propia

\section{Balance de energía eléctrica}

Se realiza un análisis sobre el balance de energía eléctrica que produce la planta GD-PV, comparada con la energía que demanda la instalación eléctrica para verificar si el sistema es auto sostenible, es decir, si la planta GD-PV produce la energía total que requiere la congeladora o la planta GD-PV sólo cubre un porcentaje de la energía eléctrica que consume y el resto la toma de la red de CFE.

La tabla 7 muestra un déficit en la producción de energía con respecto a la que energía que demanda el sistema eléctrico, es decir, que aproximadamente el $25 \%$ de la energía requerida en la Planta congeladora es la que puede cubrir la planta GD-PV y que aproximadamente el $75 \%$ de la energía que consume la instalación la adquiere de la red eléctrica de la CFE.

\begin{tabular}{|l|r|r|r|}
\hline \multicolumn{5}{c}{$\begin{array}{c}\text { Balance de energía por día } \\
\mathrm{kWh} \\
\text { Fecha }\end{array}$} & $\begin{array}{c}\mathrm{kWh} \\
\text { demandados }\end{array}$ & \multicolumn{1}{c|}{$\begin{array}{c}\text { Déficit de } \\
\mathrm{kWh}\end{array}$} \\
\hline 25-ene & 1237.758 & 301.6 & 936.158 \\
\hline 26-ene & 1269.359 & 301.6 & 967.759 \\
\hline 27-ene & 1288.242 & 301.6 & 986.642 \\
\hline 28-ene & 1322.997 & 301.6 & 1021.397 \\
\hline 29-ene & 1360.182 & 301.6 & 1058.582 \\
\hline 30-ene & 1232.068 & 301.6 & 930.468 \\
\hline 31-ene & 1190.592 & 301.6 & 888.992 \\
\hline 01-feb & 1294.765 & 301.6 & 993.165 \\
\hline
\end{tabular}

Tabla 7 Balance de energía en el sistema eléctrico de la Planta congeladora durante los días de medición

Fuente: Elaboración Propia

\section{Energía producida por la GD-PV}

La energía producida por la GD-PV, $E_{\text {prod }}$ en las 5.5 horas de radiación solar de un día solar (NREL, 2010), está dada por:

$E_{\text {prod }}=W_{\text {pico }} \cdot h r_{\text {rad }} \cdot$ piezas $_{\text {módulo PV }}$

donde $W_{\text {pico }}$ es la potencia pico de un módulo fotovoltaico en Watts, $h r_{\text {rad }}$ son las horas de radiación solar en un día, y piezas $_{\text {modulo PV }}$ representa el número de módulos fotovoltaicos incluidos en la planta de GD-PV.

Derivado de lo anterior, la energía producida por la GD-PV está dada por: 
$E_{\text {prod }}=0.260 \mathrm{~kW}(5.5 \mathrm{hr})(232$ módulos $)=$ $331.76 \mathrm{kWh} /$ día

\section{Estimación de $\mathrm{CO}_{2}$ que se evita enviar al medio ambiente debido a la GD-PV}

Dentro de un estudio de eficiencia energética, debe incluirse la cantidad de emisiones de bióxido de carbono $\left(\mathrm{CO}_{2}\right)$ que se liberan al medio ambiente debido a la generación de electricidad. En México, la Comisión Reguladora de Energía (CRE) y la Secretaría de Medio Ambiente y Recursos Naturales (SEMARNAT) estiman anualmente el Factor de Emisión del Sistema Eléctrico Nacional basado en el Artículo 12 del Reglamento de la Ley de Transición de Energía (Cámara de Diputados, 2017). El último factor publicado en el Diario Oficial de la Federación fue en el año 2017 siendo este de:

\section{$0.582 \mathrm{~kg}$ de $\mathrm{CO}_{2} / \mathrm{kWh}$}

Además, se ha considerado un factor de emisión causado por la producción de energía con módulos fotovoltaicos; en los cálculos se utilizó un modelo de análisis denominado Modelo de Emisiones Globales para Sistemas integrados (GEMIS, 2018), el cual aplica un factor de:

\section{$0.135 \mathrm{~kg}$ de $\mathrm{CO}_{2} / \mathrm{kWh}$}

Y Reich, et al. (2007), invita a elegir un valor entre un rango de emisiones entre:

\subsection{0-0.317 kg de $\mathrm{CO}_{2} / \mathrm{kWh}$}

De forma empírica escogemos el factor de GEMIS para nuestros cálculos, debido a que se encuentra dentro del rango de Reich.

Durante un mes de facturación, el total de la energía consumida por la Planta congeladora y que fue adquirida de la GD-PV es:

$$
E_{\text {cons }}=331.76 \frac{\mathrm{kWh}}{\text { día }}(30 \text { días })=9952.8 \mathrm{kWh}
$$

Entonces, las emisiones de $\mathrm{CO}_{2}$ que se evita enviar al medio ambiente $\epsilon_{\text {cons }}$ por la energía que se consume a través de la GD-PV es:

$\epsilon_{\text {cons }}=0.582 \frac{\mathrm{kg} \mathrm{CO}}{\mathrm{kWh}}(9952.8 \mathrm{kWh})=5792.53 \mathrm{~kg} \mathrm{CO} 2$
Las emisiones de $\mathrm{CO}_{2}$ producidas $\epsilon_{\text {prod }}$ causadas por la producción de energía con la GD-PV son:

$$
\epsilon_{\text {prod }}=0.135 \frac{\mathrm{kg} \mathrm{CO}}{\mathrm{kWh}}(9952.8 \mathrm{kWh})=1343.63 \mathrm{~kg} \mathrm{CO} 2
$$

Finalmente, las emisiones de $\mathrm{CO}_{2}$ netas $\epsilon_{\text {net }}$ que se evita enviar a la atmósfera es la diferencia entre $\epsilon_{\text {cons }}$ y $\epsilon_{\text {prod }}$ :

$$
\epsilon_{\text {net }}=\epsilon_{\text {cons }}-\epsilon_{\text {cons }}=5792.53 \mathrm{~kg} \mathrm{CO}-
$$$$
1343.63 \mathrm{~kg} \mathrm{CO}_{2}=4448.9 \mathrm{~kg} \mathrm{CO}
$$

Este es un dato significativo, muestra que se están dejando de emitir al medio ambiente 4.445 Toneladas de $\mathrm{CO}_{2}$, el cual es un aporte revelador para la disminución de la huella de carbono.

\section{Recomendaciones de eficiencia energética}

Las medidas de eficiencia energética que se proponen en este estudio se enumeran a continuación:

- $\quad$ Para mejorar la eficiencia de la GD-PV en épocas pico, se recomienda un reajuste en la inclinación de los módulos del sistema entre un rango de 10 a 15 grados. Se puede ganar hasta $5 \%$ de eficiencia y se evita esfuerzos mecánicos a los módulos por su propio peso y por la presión ejercida por el aire (zona de estancamiento).

- Se recomienda levantar los módulos. Tomando como referencia la parte más baja de los módulos, se debe separar del techo una distancia mínima de $50 \mathrm{~cm}$. Esta modificación favorecerá la circulación de aire para enfriar los módulos y se evitará zonas de estancamiento. Se prevé un aumento de eficiencia de un $5 \%$.

- $\quad$ Para evitar pérdidas de eficiencia de la GD-PV debido al sombreado de los módulos, se debe disminuir la barda perimetral que rodea al sistema fotovoltaico a una altura máxima de 40 $\mathrm{cm}$. Con esta acción, la eficiencia de la GD-PV aumentará un $2 \%$. 
- $\quad$ El sombreado producido por la planta GD-PV, evita que haya una ganancia térmica diaria por techo de aproximadamente $236.62 \mathrm{~kW}$ por día.

- Se debe modificar las conexiones de las cargas monofásicas en los tableros de distribución de acuerdo con la sugerencia descrita en secciones anteriores con el fin de reducir el \% desbalance entre fases y evitar circulación de corriente por el neutro que provoca daños a la instalación, a los equipos y a las personas.

- Se recomienda sustituir los motores eléctricos de $12 \mathrm{~kW}$ y $9 \mathrm{~kW}$ debido a que son muy viejos y de baja eficiencia por motores de alta eficiencia para disminuir el consumo de dichos equipos en un $5 \%$.

Instalar variadores de velocidad en los 6 congeladores de $12 \mathrm{~kW}$, para reducir los picos de potencia en los paros y arranques del compresor. Los congeladores representan la mayor carga de la GD-PV. Con esta acción se puede tener ahorros de consumo y demanda de un $15 \%$.

- Con la planta GD-PV se evita la emisión a la atmosfera de $4,448.9 \mathrm{~kg}$ de $\mathrm{CO}_{2}$ equivalente.

\section{Agradecimiento}

Los autores agradecen a la Universidad Autónoma de Campeche el apoyo y el esfuerzo brindado, durante el desarrollo de este trabajo, a sus investigadores para que difundan los temas de investigación en los cuales están inmersos.

\section{Conclusiones}

En este artículo se presenta un estudio de eficiencia energética a una empresa congeladora de pescados y mariscos que posee una planta de Generación Distribuida Fotovoltaica (GDF-PV) a través de una auditoría energética, analizándola información de las principales variables eléctricas que actúan como protagonistas en la facturación del servicio de la empresa suministradora.
Se sugieren algunas recomendaciones que requieren inversión de capital y que al implementarlas se tendrán valores significativos de ahorro y uso eficiente de la energía en el sistema eléctrico de la Planta congeladora.

Se concluye que la metodología empleada es una guía de acciones que se deben realizar de manera ordenada y con un orden cronológico para asegurar la reducción de costos de consumos (kWh) y demandas $(\mathrm{kW})$ de energía eléctrica a través de la aplicación de la eficiencia energética.

\section{Referencias}

Agustín Castejón, Germán Santamaría. (2012). Instalaciones solares fotovoltaicas. Editex. ISBN eBook 978-84-9771-749-6.

Cámara de Diputados. "Reglamento de la Ley de Transición Energética". Art. 12. Año 2017. Consultado en: http://www.diputados.gob.mx/LeyesBiblio/y/R eg_LTE_040517.pdfregle

Delgado A. J. S. Villalón, O. R. \& Sánchez, M. M. L. (2020). Análisis tecno-económico de la operación en esquema de red inteligente $\mathrm{y}$ reserve energética en usuarios conectados a paneles fotovoltaicos. Red internacional de Investigadores en Competitividad, 13, 736-756.

DOF: 07/03/2017 RESOLUCIÓN de la Comisión Reguladora de Energía por la que expide las disposiciones administrativas de carácter general, los modelos de contrato, la metodología de cálculo de contraprestación y las especificaciones técnicas generales, aplicables a las centrales eléctricas de generación distribuida y generación limpia distribuida.

GEMIS. "Modelo de Emisiones Globales para Sistemas integrados". Consultado en https://ghgprotocol.org/Third-Party-

Databases/GEMIS

Global Energy Trade, reporte marzo-abril, (2020). Monitor de información comercial $e$ indice de Precios de Generación Solar Distribuida en México. https://www.worldenergytrade.com/energiasalternativas/general/generacion-distribuidapotencial-de-crecimiento 
http://www.beta.inegi.org.mx/app/areasgeografi cas/?ag=04. INEGI (2015)

León Llanos J. A. (2020). Generación distribuida mediante el diseño de un Sistema fotovoltaico conectado a la red de distribución en el Instituto ISA-Chiclayo.

Lezama Zárraga, F. R, Andrade Durán, J. E., Ovando Sierra, J. C. \& Castillo Téllez, M. Aplicación de Eficiencia Energética para optimizar el Sistema de Iluminación en una Institución de Nivel Superior. Revista de aplicaciones de la Ingeniería. ISSN 2523-6873. Edit. Ecorfan Bolivia, Vol. 2, No. 8, páginas 2734. 2018.

Luis C. A., A. R., \& María Beatriz, P. (2020). Proyectos fotovoltaicos en generación distribuida. ¿Rentabilidad Privada o Sustentabildad Ambiental? Revista politécnica, 45(2), 31-40.

Mayra Robles López et al. La eficiencia energética en microempresas mexicanas. https://www.uv.mx/iiesca/files/2017/03/19CA20 1602.pdf

Merle Potter, David Wiggert, Bassem Ramadam, (2012). Mecánica de Fluidos $4^{a}$ edición. Editorial CENGAGE Learning, ISBN 13:978-607-519-450-9.

N.H. Reich, E.A. Alsema, W.G.J.H.M. van Sark, E. Nieuwlaar. " $\mathrm{CO}_{2}$ Emissions of $\mathrm{PV}$ in the perspective of a renewable energy economy". $22^{\text {nd }}$ European Photovoltaic Solar Energy Conference, pp. 3538-3542. Milan, Italy. September 2007.

Ramos, E. (2020). La generación distribuida: el camino hacia la producción descentralizada de electricidad y pautas para su reglamentación. Forseti. Revista de derecho, 8(11), 07-35.

Rayenari D. C., J. Chan-González, Roger C., Jesús Contreras, M. Margarita Castillo-Téllez, Francisco Lezama, Víctor Lanz, J. Ortega (2015) Sistema fotovoltaico interconectado a red instalado en el estacionamiento de la Facultad de Ingeniería de la Universidad Autónoma de Campeche. Memorias del Congreso Nacional de Energía Solar.
Terol Calvo, S. (2020) El acceso de la energía distribuida en conjuntos residenciales. Evaluación de sistemas fotovoltaicos comunitarios en la villa olímpica (Doctoral dissertation).

U.S. Department of Energy. National Renewable Energy Laboratory, (2010). https://maps.nrel.gov/rede-

mexico/?aL=wn05da $\% 255 \mathrm{Bv} \% 255 \mathrm{D} \% 3 \mathrm{Dt} \% 26$ u-o1v1\%255Bv\%255D\%3Dt\%26u-

o1v1\%255Bd\%255D\%3D1\%26mwBW5c\%25 5Bv\%255D\%3Dt\%26mwBW5c\%255Bd\%255 D\%3D2\%26mwBW5c\%255Br\%255D\%3Dt\%2 6tpot_result_mexico_145835\%255Bv\%255D\% 3Dt\%26tpot_result_mexico_145835\%255Bd\% 255D\%3D3\%26tpot_result_mexico_145835\%2 $55 \mathrm{Br} \% 255 \mathrm{D} \% 3 \mathrm{Dt} \& \mathrm{bL}=\mathrm{clight} \& \mathrm{cE}=0 \& 1 \mathrm{R}=0 \&$ $\mathrm{mC}=21.861498734372567 \% 2 \mathrm{C}-$ $87.4951171875 \& z L=5$

Víctor Lanz Gutierrez de Velazco, Manuel Cervera, (2018) Auditorías Energéticas a las Instalaciones de la Universidad Autónoma de Campeche, Comunicado Personal.

Victorio Santiago Díaz, Raúl Oscar. (2005) Acondicionamiento termico de edificios/ Thermal Preparation of Buildings. Editorial NOBUKO, ISBN 987-1135-94-7.

Yunus Cengel, Afshin J. Ghajar, (2011) Transferencia de Calor y Masa $4^{a}$ edición. Editorial McGraw Hill, ISBN 978-607-150540-8. 